\title{
Correspondence
}

\section{Comments on "On Resistor-Induced Thermal Noise in Linear Circuits"}

Manuel Vargas and Ramon Pallàs-Areny

In a recent paper [1], Giannetti considered the thermal noise generated by a resistor $R$ on a generic output port in any linear network. He reasoned that, because the contribution of the thermal noise of the resistor to the output noise of the circuit vanishes for both $R \rightarrow 0$ and $R \rightarrow \infty$, there should be a resistor value whose contribution to the output noise is maximal. In order to find that value, from Fig. 1 he obtained that the thermal noise contributed by $R$ was [1, (3)]

$$
\overline{v_{u}^{2}}(R)=\left|H^{\prime}\right|^{2}\left|\frac{R}{R+Z}\right|^{2} \frac{4 k T}{R}=A\left|\frac{R}{R+Z}\right|^{2} \frac{1}{R}
$$

which is incorrect because the second and third terms of (1) do not have the dimensions of voltage spectral density. The correct analysis yields

$$
\overline{v_{u}^{2}}(R)=\left|H^{\prime}\right|^{2}\left|\frac{R Z}{R+Z}\right|^{2} \frac{4 k T}{R}=A\left|\frac{R Z}{R+Z}\right|^{2} \frac{1}{R} .
$$

The maximal noise will result for that value of $R$ that nulls the first derivative of (2), which is $R=|Z|$. This agrees with [1, (5)] because the mistake in (1) reduces to a multiplying factor $(Z)$ that does not depend on $R$. The maximal output noise contributed by the thermal noise of $R$ can then be calculated by replacing $R$ with $|Z|$ in (2) to obtain

$$
\overline{v_{u}^{2}}=\frac{2 k T|Z|\left|H^{\prime}\right|^{2}}{1+\cos \varphi}
$$

which is different from that given by [1, eq. (6)] [derived from (1)], whose dimensions were not those of a voltage spectral density and included $H^{\prime}(s)$ instead of $\left|H^{\prime}\right|$. Nevertheless, the conclusion in [1] is correct; the output voltage spectral density will increase if the impedance $Z$ is not purely resistive.

This interesting result has no simple explanation and deserves further elaboration because it might lead to the wrong conclusion that a reactive impedance $Z$ would increase the output noise. According to (3), the voltage spectral density at the output of an amplifier whose input impedance includes, say, a shunting capacitance, will certainly increase when $R=|Z|$, but, because of the frequency dependence of the impedance of a capacitance, that condition will happen at a single frequency. What will then happen in a finite frequency bandwidth? If $Z=R^{*}+j X^{*}$, the output noise power from $f_{L}$ to $f_{H}$ will be

$$
\begin{aligned}
V_{u}^{2} & =\int_{f_{L}}^{f_{H}} \overline{v_{u}^{2}} d f \\
& =4 k T R \int_{f_{L}}^{f_{H}}\left|\frac{Z}{R+Z}\right|^{2}\left|H^{\prime}\right|^{2} d f \\
& =4 k T R \int_{f_{L}}^{f_{H}} \frac{R^{* 2}+X^{* 2}}{\left(R+R^{*}\right)^{2}+X^{* 2}}\left|H^{\prime}\right|^{2} d f
\end{aligned}
$$

whose result is determined by the frequency dependence of $R^{*}, X^{*}$, and $H^{\prime}$. For the sake of simplicity, let us assume that $\left|H^{\prime}\right|=1$. For a

Manuscript received March 21, 2002; revised March 21, 2002.

The authors are with the Departament d'Enginyeria Electrònica, Universitat Politècnica de Catalunya, Barecelona, Spain (e-mail: elerpa@eel.upc.es).

Publisher Item Identifier S 0018-9456(02)04873-8.

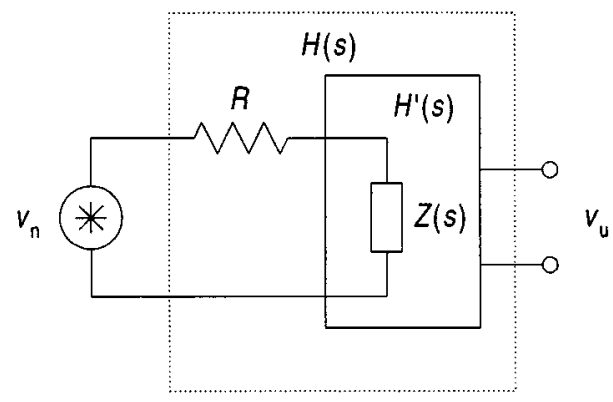

Fig. 1. Effect of the thermal noise of a resistor in a circuit can be analyzed by separating the resistor from the rest of that circuit.

purely capacitive input impedance $\left(R^{*}=0,1 / X^{*}=-2 \pi f C\right)$, we would have

$$
\left.V_{u}^{2}\right|_{R^{*}=0}=\frac{k T}{C} \frac{2}{\pi} \arctan \frac{2 \pi\left(f_{H}-f_{L}\right) R C}{1+\left(2 \pi \sqrt{f_{H} f_{L}} R C\right)^{2}}
$$

whose maximal value would be

$$
\left.V_{u(\max )}^{2}\right|_{R^{*}=0}=4 k T R \sqrt{f_{L} f_{H}} \arctan \frac{f_{H}-f_{L}}{2 \sqrt{f_{H} f_{L}}}
$$

at

$$
R=\left|Z\left(j 2 \pi \sqrt{f_{L} f_{H}}\right)\right|=\frac{1}{2 \pi \sqrt{f_{L} f_{H}} C} .
$$

For a purely resistive input impedance $\left(Z=R^{*}\right)$, we would have

$$
\left.V_{u}^{2}\right|_{Z=R^{*}}=4 k T R\left(\frac{R^{*}}{R+R^{*}}\right)^{2}\left(f_{H}-f_{L}\right)
$$

whose maximal value would be

$$
\left.V_{u(\max )}^{2}\right|_{Z=R^{*}}=k T R\left(f_{H}-f_{L}\right)
$$

at $R=R^{*}$.

Dividing (6) by (9) yields

$$
\frac{\left.V_{u(\max )}^{2}\right|_{R^{*}=0}}{\left.V_{u(\max )}^{2}\right|_{Z=R^{*}}}=\frac{4 \sqrt{f_{L} f_{H}}}{f_{H}-f_{L}} \arctan \frac{f_{H}-f_{L}}{2 \sqrt{f_{H} f_{L}}} .
$$

When $f_{H}-f_{L} \ll \sqrt{f_{H} f_{L}}$, the output noise power ratio is 2 . When $f_{H}-f_{L} \gg \sqrt{f_{H} f_{L}}$, the output noise power ratio is

$$
\frac{\left.V_{u(\max )}^{2}\right|_{R^{*}=0}}{\left.V_{u(\max )}^{2}\right|_{Z=R^{*}}} \cong \frac{4 \sqrt{f_{L} f_{H}}}{f_{H}-f_{L}} \frac{\pi}{2}=\frac{2 \pi \sqrt{f_{L} f_{H}}}{f_{H}-f_{L}} \ll 1 .
$$

Therefore, from the point of view of output noise, when the source resistance and the input impedance are matched $(R=|Z|)$, for narrowband signals it is advisable to reduce the input capacitance as much as possible, whereas for a wide band signal, the input capacitance helps in reducing the output noise power. In the usual cases of voltage or current measurement we have $R \ll|Z|$ and $R \gg|Z|$, respectively, which results in minimal output voltage noise spectral density. By the same token, the output noise contributed by the amplifier input resistance $R^{*}$ will be small whenever it is unmatched to $R$. Thus, the common design practice to reduce voltage or current loading also reduces output noise.

\section{REFERENCES}

[1] R. Giannetti, "On resistor-induced thermal noise in linear circuits," IEEE Trans. Instrum. Meas., vol. 49, pp. 87-88, Feb. 2000. 